\title{
Euler-Boole Summation Revisited
}

\section{Jonathan M. Borwein, Neil J. Calkin, and Dante Manna}

1. INTRODUCTION. The Euler-Maclaurin summation formula [8, 2.11.1],

$$
\begin{aligned}
\sum_{j=a}^{n-1} f(j)= & \int_{a}^{n} f(x) d x+\sum_{k=1}^{m} \frac{B_{k}}{k !}\left(f^{(k-1)}(n)-f^{(k-1)}(a)\right) \\
& +\frac{(-1)^{m+1}}{m !} \int_{a}^{n} \widetilde{B}_{m}(y) f^{(m)}(y) d y,
\end{aligned}
$$

is a well-known formula from classical analysis giving a relation between the finite sum of values of a function $f$, whose first $m$ derivatives are absolutely integrable on $[a, n]$, and its integral, for $a, m, n \in \mathbb{N}, a<n$. This elementary formula appears often in introductory texts $[2,17]$, usually in reference to a particular applicationStirling's asymptotic formula. However, general approaches to such formulae are not often mentioned in the same context.

In the formula above, the $B_{l}$ are the Bernoulli numbers and the $\widetilde{B}_{l}(x)$ are the periodic Bernoulli polynomials. The Bernoulli polynomials are most succinctly characterized by a generating function $[1,23.1 .1]$ :

$$
\frac{t e^{x t}}{e^{t}-1}=\sum_{n=0}^{\infty} B_{n}(x) \frac{t^{n}}{n !}
$$

The periodic Bernoulli polynomials are defined by taking only the fractional part of $x$ : $\widetilde{B}_{n}(x):=B_{n}(\{x\})[8,24.2 .11-12]$. Evaluating at the point $x=0$ gives the Bernoulli numbers $[1,23.1 .2]: B_{l}:=B_{l}(0)$.

A similar formula comes from starting with a different set of polynomials. The Euler polynomials $E_{n}(x)$ are given by the generating function $[1,23.1 .1]$

$$
\frac{2 e^{x t}}{e^{t}+1}=\sum_{n=0}^{\infty} E_{n}(x) \frac{t^{n}}{n !} .
$$

Let the periodic Euler polynomials $\widetilde{E}_{n}(x)$ be defined by $\widetilde{E}_{n}(x+1)=-\widetilde{E}_{n}(x)$ and

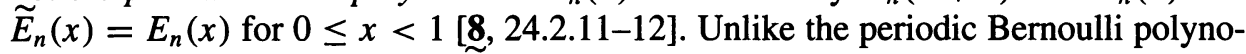
mials, which have period 1, the $\widetilde{E}_{n}(x)$ have period 2. Thirdly, define the Euler numbers by $E_{n}:=2^{n} E_{n}(1 / 2)[1,23.1 .2]$; i.e., $[8,24.2 .6]$,

$$
\frac{2 e^{t}}{e^{2 t}+1}=\sum_{n=0}^{\infty} E_{n} \frac{t^{n}}{n !} .
$$

The alternating version of (1), using Euler polynomials, is the Boole summation formula [8, 24.17.1-2]: Let $a, m, n \in \mathbb{N}, a<n$. If $f(t)$ is a function on $t \in[a, n]$ 
with $m$ absolutely integrable derivatives, then for $0<h<1$,

$$
\begin{aligned}
\sum_{j=a}^{n-1}(-1)^{j} f(j+h)= & \frac{1}{2} \sum_{k=0}^{m-1} \frac{E_{k}(h)}{k !}\left((-1)^{n-1} f^{(k)}(n)+(-1)^{a} f^{(k)}(a)\right) \\
& +\frac{1}{2(m-1) !} \int_{a}^{n} f^{(m)}(x) \widetilde{E}_{m-1}(h-x) d x .
\end{aligned}
$$

The first appearance of this formula is due to Boole [4]; a similar formula is believed [9] to have been known to Euler as well. Mention of this beautiful formula in the literature is regrettably scarce in comparison to that of (1), although in [5] it is used to explain a curious property of truncated alternating series for $\pi$ and $\log 2$-for which Boole summation is better suited than Euler-Maclaurin.

In his 1960 note, Strodt [15] indicated a unified operator-theoretic approach to proving both of these formulae, but with very few of the details given. In the years since, excellent generalizations of Euler-Maclaurin summation have appeared [3], yet not using this particular approach. The main goals of this note are to fill in Strodt's details, and to demonstrate the extent to which his ideas can be extended to other cases.

We begin with a characterization of the operators suggested by Strodt and their associated polynomials. Section 2 ends with a couple of main theorems about these polynomials, which lead to corollaries concerning both the Euler and Bernoulli polynomials - this is the content of Section 3. The following section describes the polynomials arising from well-known probability densities. In Section 5 we detail Strodt's unified development of the summation formulae of Euler and Boole. Section 6 describes a generalized development of inversion formulae involving the Euler and Bernoulli numbers. We conclude our discussion with a conjecture and proof of a general asymptotic formula in Section 7.

2. STRODT'S OPERATORS AND POLYNOMIALS. We introduce a class of operators on functions of a finite real interval. Let $C[\mathbb{R}]$ denote the continuous functions on the real line in the supremum norm. Define, for $n \in \mathbb{N}$, the uniform interpolation Strodt operators $\mathcal{S}_{n}: C[\mathbb{R}] \mapsto C[\mathbb{R}]$ by

$$
\mathcal{S}_{n}(f)(x):=\sum_{j=0}^{n-1} \frac{1}{n} \cdot f\left(x+\frac{j}{n-1}\right)
$$

The operators used by Strodt to prove Euler-Maclaurin summation and Boole summation are both covered under this definition, as we shall demonstrate. Since the operators defined by (6) are positive, they are necessarily bounded linear operators.

We will show that an operator in this class will send a polynomial in $x$ to another one of the same degree. Furthermore, we claim they are bijections on the set of degree$k$ polynomials. This fact will be used to define both Euler and Bernoulli polynomials as inverse images of $x^{k}$ under different operators. There is evidence that this approach is known, at least informally, in the Bernoulli case [18], but not for Euler polynomials. mials.

We begin by noting an important property of any interpolation operator on polyno-

Proposition 2.1. For each $k \in \mathbb{N}$, let $P_{k}:=\left\{\sum_{i=0}^{k} a_{i} x^{i}: a_{i} \in \mathbb{R}\right\} \cong \mathbb{R}^{k+1}$. Then for all $n \in \mathbb{N}$, given $A \in P_{k}$ there is a unique $B \in P_{k}$ such that $\mathcal{S}_{n}(B)=A$. 
This property will be proven for a more general class of operators. Given a finite set of points $\left\{x_{i}\right\}_{i=1}^{N} \subset[0,1]$ and a probability weight function

$$
w:\left\{x_{i}\right\}_{i=1}^{N} \rightarrow(0,1) \text { so that } \sum_{i=1}^{N} w\left(x_{i}\right)=1,
$$

we define the corresponding finite Strodt operator

$$
\mathcal{S}_{w}(f)(x):=\sum_{i=1}^{N} f\left(x+x_{i}\right) w\left(x_{i}\right)
$$

that generalizes $\mathcal{S}_{n}$. Even more broadly, define the generalized Strodt operators

$$
\mathcal{S}_{\mu}(f)(x):=\int f(x+u) d \mu,
$$

where the integral is a Lebesgue-Stieltjes integral [16, pp. 282-284] with measure $\mu$, taken over some measurable subset of $u \in \mathbb{R}$. We will often consider the special case $d \mu=g(u) d u$ where $g(u)$ is any absolutely continuous probability weight function with finite moments:

$$
\int g(u) d u=1 \text { and } \int|u|^{k} g(u) d u<\infty \text { for all } k \in \mathbb{N} .
$$

Here and from this point forward we will use the convention $\int=\int_{-\infty}^{\infty}$, where the support of $g$ is used to effectively limit the interval. Also, we will use $\mathcal{S}_{g}$ in place of $\mathcal{S}_{\mu}$ in this case.

If $\mu$ is a discrete Lebesgue measure, we formally write $g$ as a sum of weighted point masses. In the finite case,

$$
\mathcal{S}_{w}=\mathcal{S}_{g}, \quad \text { where } g(u)=\sum_{i=1}^{N} w\left(x_{i}\right) \delta_{x_{i}}(u) .
$$

This is so we can use the notation $\mathcal{S}_{g}(f)$ in each of the above cases. We will prove that a version of Proposition 2.1 holds for even this most general class of operators.

Now suppose $f \in P_{k}$; that is, $f:=\sum_{n=0}^{k} f_{n} x^{n}$, for $f_{n} \in \mathbb{R}$. By definition,

$$
h(x):=\mathcal{S}_{g}(f)=\int \sum_{n=0}^{k} f_{n}(x+u)^{n} g(u) d u,
$$

and we see via the binomial theorem that $h(x)$ is again a polynomial of degree $k$ :

$$
h(x)=\sum_{j=0}^{k} h_{j} x^{j}
$$

where $h_{j}=\sum_{n=j}^{k} f_{n}\left(\begin{array}{l}n \\ j\end{array}\right) M_{n-j}$ and

$$
M_{l}:=\int u^{l} g(u) d u
$$


Regarding degree- $k$ polynomials as vectors of length $k+1$ via the natural isomorphism, we see that the restriction of the operator $\mathcal{S}_{g}$ to $P_{k} \cong \mathbb{R}^{k+1}$ can be represented by a $(k+1) \times(k+1)$ matrix. The coefficients of this matrix are read directly from (12):

$$
\left.\mathcal{S}_{g}\right|_{P_{k}}[i, j]:= \begin{cases}\left(\begin{array}{c}
j-1 \\
i-1
\end{array}\right) M_{j-i} & \text { for } 1 \leq i \leq j \leq k+1 \\
0 & \text { otherwise. }\end{cases}
$$

This allows us to prove that this operator is invertible.

Proposition 2.2. Let $g$ be a probability density function whose absolute moments exist. For all $h \in P_{k}$, there is a unique $f \in P_{k}$ so that $\mathcal{S}_{g}(f)=h$.

Proof. We see from (14) that $\left.\mathcal{S}_{g}\right|_{P_{k}}$ is an upper-triangular matrix whose determinant is

$$
\operatorname{det}\left(\left.\mathcal{S}_{g}\right|_{P_{k}}\right)=\prod_{t=0}^{k}\left(\begin{array}{l}
t \\
t
\end{array}\right) M_{0}=1
$$

and so $h(x)$ is the unique solution of a linear system of equations:

$$
h=\mathcal{S}_{g}^{(-1)} f \text {. }
$$

The reader will note that Proposition 2.1 follows from this as a special case.

We can recover both the Euler polynomials and the Bernoulli polynomials using this uniqueness property with different weight functions. Define the Euler operator as

$$
\mathcal{S}_{E}(f)(x):=\frac{f(x)+f(x+1)}{2} ; \quad \text { i.e., } g(u):=\delta_{0}(u) / 2+\delta_{1}(u) / 2 ;
$$

and the Bernoulli operator by

$$
\mathcal{S}_{B}(f)(x):=\int_{0}^{1} f(x+u) d u ; \quad \text { i.e., } g(u):=\chi_{[0,1]} .
$$

We now claim that the set of Euler polynomials $E_{n}(x)$ is the unique set of polynomials that satisfy

$$
\mathcal{S}_{E}\left(E_{n}(x)\right)=x^{n} \quad \text { for all } n \in \mathbb{N}_{0},
$$

while the Bernoulli polynomials are the unique polynomials which satisfy

$$
\mathcal{S}_{B}\left(B_{n}(x)\right)=x^{n} \quad \text { for all } n \in \mathbb{N}_{0}
$$

Remark 1. One now sees the motivation behind the definition of $\mathcal{S}_{n}$ in (6). The Euler operator is $\mathcal{S}_{2}$. The Bernoulli operator corresponds via a Riemann sum to the limit of $\mathcal{S}_{n}$ as $n \rightarrow \infty$. Hence, the range of $n$ provides an interpolation of sorts between these two developments.

We shall prove that the generating function characterizations of the Bernoulli and Euler polynomials follow from (19) and (20). Thus these formulae comprise sufficent 
definitions of the polynomials. Unlike the conventional recursive definitions of $B_{n}(x)$ and $E_{n}(x)$, these definitions require no extra conditions, such as initial values [15]. Furthermore, they each take the form $\mathcal{S}_{g}^{-1}\left(x^{n}\right)$ for different weight functions. This suggests a natural generalization of Bernoulli and Euler polynomials which we will call Strodt polynomials. They will be denoted $P_{n}^{g} \equiv P_{n}, n \in \mathbb{N}_{0}$ (we will typically suppress the $g$ in the notation).

Theorem 2.3. For each $n$ in $\mathbb{N}_{0}$, let $P_{n}^{g}(x)$ be the Strodt polynomial associated with a given density $g(x)$; that is, for all $x \in \mathbb{R}, P_{n}^{g}(x)$ is defined implicitly by the relation

$$
\mathcal{S}_{g}\left(P_{n}^{g}(x)\right)=x^{n} \quad \text { for all } n \in \mathbb{N}_{0},
$$

where $\mathcal{S}_{g}$ is a Strodt operator. Then

$$
\frac{d}{d x} P_{n}^{g}(x)=n P_{n-1}^{g}(x) \text { for all } n \in \mathbb{N} .
$$

Proof. By the Lebesgue Dominated Convergence Theorem [13], we have that

$$
\begin{aligned}
\frac{d}{d x} \int P_{n}(x+u) g(u) d u & =\lim _{\nu \rightarrow \infty} \int v\left(P_{n}\left(x+\frac{1}{v}+u\right)-P_{n}(x+u)\right) g(u) d u \\
& =\int \lim _{\nu \rightarrow \infty} v\left(P_{n}\left(x+\frac{1}{v}+u\right)-P_{n}(x+u)\right) g(u) d u \\
& =\int \frac{d}{d x} P_{n}(x+u) g(u) d u .
\end{aligned}
$$

Therefore, in view of (21), we have that

$$
\mathcal{S}_{g}\left(\frac{d}{d x} P_{n}-n P_{n-1}\right)=\frac{d}{d x}\left(x^{n}\right)-n x^{n-1}=0 .
$$

But $\mathcal{S}_{g}$ is one-to-one on polynomials by Proposition 2.2, hence the desired conclusion follows.

We note that the class of Strodt polynomials comprises a subset of Appell sequences [12]. These are the polynomial sequences given by generating functions of the form

$$
\sum_{n=0}^{\infty} A_{n}(x) \frac{t^{n}}{n !}=\frac{e^{x t}}{G(t)} .
$$

Here $G(t)$ is a function defined formally by the coefficient sequence of its series in $t$, where the only restriction is that the leading term must not be zero. This is in fact equivalent to (22) [12].

The next theorem clarifies the relation between Strodt polynomials and Appell sequences.

Theorem 2.4. Suppose a class of polynomials $\left\{C_{n}(x)\right\}_{n \geq 0}$ with real coefficients has an exponential generating function of the form

$$
\sum_{n=0}^{\infty} C_{n}(x) \frac{t^{n}}{n !}=e^{x t} R(t),
$$


where $R(t)$ is a continuous function on the real line. Then the exponential generating function of the polynomial sequence which is the image of the $C_{n}$ under the operator $\mathcal{S}_{g}$ is given by

$$
\sum_{n=0}^{\infty} \mathcal{S}_{g}\left(C_{n}(x)\right) \frac{t^{n}}{n !}=e^{x t} R(t) Q_{g}(t)
$$

where

$$
Q_{g}(t):=\int e^{u t} g(u) d u
$$

Proof. Assume that the parameter $t$ is within the radius of uniform convergence of the formal power series (25) for an arbitrary fixed value of $x$. Then we can integrate the series (25) termwise to produce:

$$
\sum_{n=0}^{\infty} \int C_{n}(x+u) g(u) d u \frac{t^{n}}{n !}=\int e^{(x+u) t} R(t) g(u) d u .
$$

This is equivalent to (26).

As in the case of the $P_{n}^{g}(x)=P_{n}(x)$, the functions $Q_{g}(t)=Q(t)$ are implicitly dependent on the weight function $g(u)$, but this will be suppressed in the notation.

Expanding the exponential integrand of (27) in a Taylor series, and then integrating termwise, we see that

$$
Q(t)=\sum_{n=0}^{\infty} M_{n} \frac{t^{n}}{n !}
$$

where $M_{n}$ is, as in (13), the $n$th moment of the cumulative distribution function of the density $g(u)$. Therefore $Q(t)$ is the moment generating function of $g$. See [6] for more information on moment generating functions.

Theorem 2.4 has the following consequence on the generating function of Strodt polynomials:

$$
\sum_{n=0}^{\infty} P_{n}(x) \frac{t^{n}}{n !}=\frac{e^{x t}}{Q(t)}
$$

To see why this must be true, apply (26) with $C_{n}(x)=P_{n}(x)$, and conclude that $R(t)$ must equal $1 / Q(t)$.

Remark 2. Now we see the precise connection to Appell sequences: the Appell sequence whose generating function comes in the form $e^{x t} / Q(t)$ is a Strodt polynomial sequence exactly when $Q(t)$ is the moment generating function of some cumulative distribution function.

We can now verify our claim regarding the definition of Bernoulli and Euler polynomials.

Corollary 2.5. Formulae (19) and (20) are sufficient definitions of the classical Euler polynomials and Bernoulli polynomials, respectively. 
Proof. We show that the conditions (19) and (20) imply the generating function characterization of the two classes of polynomials. Start with the Euler polynomials, whose generating function is given by $[8,24.2 .8]$ as

$$
\sum_{n=0}^{\infty} E_{n}(x) \frac{t^{n}}{n !}=\frac{2 e^{x t}}{e^{t}+1}
$$

The Euler polynomials satisfy

$$
\sum_{n=0}^{\infty} \mathcal{S}_{E}\left(E_{n}(x)\right) \frac{t^{n}}{n !}=\frac{2 e^{x t}}{e^{t}+1} \cdot Q_{E}(t)
$$

by Theorem 2.4 . We verify that

$$
Q_{E}(t)=\int e^{u t}\left(\frac{\delta_{0}(u)}{2}+\frac{\delta_{1}(u)}{2}\right) d u=\frac{e^{t}+1}{2},
$$

and so

$$
\sum_{n=0}^{\infty} \mathcal{S}_{E}\left(E_{n}(x)\right) \frac{t^{n}}{n !}=e^{x t}=\sum_{n=0}^{\infty} x^{n} \frac{t^{n}}{n !}
$$

Therefore, by uniqueness of power series coefficients, we have that for each integer $n \geq 0, E_{n}(x)$ is a polynomial of degree $n$ that satisfies (19); that is,

$$
\mathcal{S}_{E}\left(E_{n}(x)\right)=x^{n} \quad \text { for all } n \in \mathbb{N}_{0}
$$

Proposition 2.2 assures us that the $E_{n}(x)$ are completely determined by this property, so it can be used as a definition.

The argument for Bernoulli polynomials is similar. This time,

$$
Q_{B}(t)=\int_{0}^{1} e^{u t} d u=\frac{e^{t}-1}{t} .
$$

Using the generating function for Bernoulli polynomials,

$$
\sum_{n=0}^{\infty} B_{n}(x) \frac{t^{n}}{n !}=\frac{t e^{x t}}{e^{t}-1}
$$

Theorem 2.4 implies that

$$
\sum_{n=0}^{\infty} \mathcal{S}_{B}\left(B_{n}(x)\right) \frac{t^{n}}{n !}=\frac{t e^{x t}}{e^{t}-1} \cdot \frac{e^{t}-1}{t}=e^{x t}=\sum_{n=0}^{\infty} x^{n} \frac{t^{n}}{n !} .
$$

Thus, by uniqueness of power series coefficients, the $B_{n}(x)$ are degree- $n$ polynomials satisfying

$$
\mathcal{S}_{B}\left(B_{n}(x)\right)=x^{n} \quad \text { for all } n \in \mathbb{N}_{0} .
$$

By Proposition 2.2, the Bernoulli polynomials are the unique polynomials that satisfy this property. 
3. FIRST CONSEQUENCES. We now show a few interesting special cases of Strodt polynomials and add to the list of properties that are directly implied by Theorems 2.3 and 2.4. In addition to what is shown here, we believe that most properties of Bernoulli and Euler polynomials that appear as formulas in a reference such as [1] or [8] are true in general for Strodt polynomials.

The Bernoulli polynomials of the kth order (or type, but not to be confused with the Bernoulli polynomials of the $k$ th $k i n d$, which are altogether different!) are given by the generating function $[8,24.16 .1]$

$$
\sum_{n=0}^{\infty} B_{n}^{(k)}(x) \frac{t^{n}}{n !}=\left(\frac{t}{e^{t}-1}\right)^{k} e^{x t}
$$

We see that, for $k=1$, this generating function agrees with (2). Therefore Bernoulli polynomials of the first order are the same as regular Bernoulli polynomials: $B_{n}^{(1)}(x)=$ $B_{n}(x)$. Since the generating function of $k$ th-order Bernoulli polynomials is just $e^{x t}$ divided by a power of $Q(t)$, we can also define these polynomials by iterations of $\mathcal{S}_{B}$, the Bernoulli operator. Henceforth we will let $\mathcal{S}_{g}^{(k)}$ denote the $k$-fold composition of the Strodt operator.

Corollary 3.1. For each positive integer $k$, if a degree-n polynomial $B_{n}^{(k)}(x)$ satisfies

$$
\mathcal{S}_{B}^{(k)}\left[B_{n}^{(k)}(x)\right]=x^{n} \quad \text { for } n \in \mathbb{N}_{0}
$$

where $\mathcal{S}_{B}$ is the Bernoulli operator given in (18), then $B_{n}^{(k)}(x)$ is a Bernoulli polynomial of the kth order.

Proof. We emulate the proof of Corollary 2.5 for Bernoulli polynomials. Use Theorem 2.4 inductively on $k \geq 1$ to show that the following is true for Bernoulli polynomials of the $k$ th order:

$$
\sum_{n=0}^{\infty} \mathcal{S}_{B}^{(k)}\left[B_{n}^{(k)}(x)\right] \frac{t^{n}}{n !}=\left(\frac{t}{e^{t}-1}\right)^{k} e^{x t}\left(\frac{e^{t}-1}{t}\right)^{k}=\sum_{n=0}^{\infty} x^{n} \frac{t^{n}}{n !}
$$

Thus, with $k$ fixed, for each $n \geq 1, B_{n}^{(k)}(x)$ is a degree- $n$ polynomial satisfying (41). An easy inductive argument with Proposition 2.2 can be used to show that the $B_{n}^{(k)}(x)$ are uniquely defined by this property.

A similar property holds for Euler polynomials of the kth order.

Corollary 3.2. Let $0 \leq k, n \in \mathbb{N}$. If a degree-n polynomial $E_{n}^{(k)}(x)$ satisfies

$$
\mathcal{S}_{E}^{(k)}\left[E_{n}^{(k)}(x)\right]=x^{n}
$$

where $\mathcal{S}_{E}$ is the Bernoulli operator given in (17), then $E_{n}^{(k)}(x)$ is an Euler polynomial of the kth order.

Proof. The proof is similar to that of the Bernoulli polynomials of the $k$ th order. The generating function for Euler polynomials of the $k$ th order $[8,24.16 .2]$ is

$$
\sum_{n=0}^{\infty} E_{n}^{(k)}(x) \frac{t^{n}}{n !}=\left(\frac{2}{e^{t}+1}\right)^{k} e^{x t}
$$


Fix $k \geq 1$, and use Theorem 2.4 inductively to show that

$$
\sum_{n=0}^{\infty} \mathcal{S}_{E}^{(k)}\left[E_{n}^{(k)}(x)\right] \frac{t^{n}}{n !}=\left(\frac{2}{e^{t}+1}\right)^{k} e^{x t}\left(\frac{e^{t}+1}{2}\right)^{k}=\sum_{n=0}^{\infty} x^{n} \frac{t^{n}}{n !}
$$

Therefore, by the uniqueness of power series coefficients, for each $n \geq 1, E_{n}^{(k)}(x)$ is a degree- $n$ polynomial satisfying (43). Finally, one argues inductively on $k$, using Proposition 2.2 , that the $E_{n}^{(k)}(x)$ are uniquely defined by this property.

We are motivated to create a new definition: For positive integers $n$ and $k$, the Strodt polynomials of the kth order, denoted $P_{n}^{(k)}(x)$, are the polynomials which satisfy

$$
\mathcal{S}_{g}^{(k)}\left(P_{n}^{(k)}(x)\right)=x^{n}
$$

By Proposition 2.2 they are well-defined, and arguments similar to those directly above construct generating functions of the form

$$
\sum_{n=0}^{\infty} P_{n}^{(k)}(x) \frac{t^{n}}{n !}=\frac{e^{x t}}{[Q(t)]^{k}}
$$

We now construct a binomial recurrence formula for Strodt polynomials. This is meant to generalize entry 23.1 .7 in [1], which states that

$$
B_{n}(x+h)=\sum_{k=0}^{n}\left(\begin{array}{l}
n \\
k
\end{array}\right) B_{k}(x) h^{n-k}
$$

and

$$
E_{n}(x+h)=\sum_{k=0}^{n}\left(\begin{array}{l}
n \\
k
\end{array}\right) E_{k}(x) h^{n-k} .
$$

This property is an equivalent definition of Appell sequences [12].

Corollary 3.3. For $n \in \mathbb{N}_{0}$, let $P_{n}(x)$ be a Strodt polynomial for a given weight function $g(u)$; i.e., for each $n \geq 0, P_{n}(x)$ is the unique polynomial satisfying $\mathcal{S}_{g}\left(P_{n}(x)\right)=$ $x^{n}$. Then

$$
P_{n}(x+h)=\sum_{k=0}^{n}\left(\begin{array}{l}
n \\
k
\end{array}\right) P_{k}(x) h^{n-k}
$$

Proof. Since $\mathcal{S}_{g}\left(P_{n}(x)\right)=x^{n}$, it is not hard to show using the definition of $\mathcal{S}_{g}$ that

$$
\mathcal{S}_{g}\left(P_{n}(x+h)\right)=(x+h)^{n} .
$$

Now use the binomial theorem to expand the right-hand side in powers of $x$ and $h$. But each power $x^{k}$ is equal to $\mathcal{S}_{g}\left(P_{k}(x)\right)$, again by definition. Therefore, we have

$$
\mathcal{S}_{g}\left(P_{n}(x+h)\right)=\mathcal{S}_{g}\left(\sum_{k=0}^{n}\left(\begin{array}{l}
n \\
k
\end{array}\right) P_{k}(x) h^{n-k}\right)
$$


by linearity of $\mathcal{S}_{g}$. Now apply $\mathcal{S}_{g}^{(-1)}$ to both sides, invoking Proposition 2.2, to arrive at (50).

The known recurrence formulae for Bernoulli and Euler polynomials can be derived directly from here. For example, when $h=1$, we have

$$
P_{n}(x+1)-P_{n}(x)=\sum_{k=0}^{n-1}\left(\begin{array}{l}
n \\
k
\end{array}\right) P_{k}(x) .
$$

Rewriting the left-hand side as an integral yields

$$
P_{n}(x+1)-P_{n}(x)=\int_{0}^{1} P_{n}^{\prime}(x+u) d u=n \int_{0}^{1} P_{n-1}(x+u) d u
$$

Now suppose that $g(u):=\chi_{[0,1]}$, so that the $P_{n}(x)$ are Bernoulli polynomials. Then we have that

$$
n \int_{0}^{1} B_{n-1}(x+u) d u=n \mathcal{S}_{B}\left(B_{n-1}(x)\right)=n x^{n-1} .
$$

We have thus derived a standard binomial relation for Bernoulli polynomials [8, 24.5.1]:

$$
n x^{n-1}=\sum_{k=0}^{n-1}\left(\begin{array}{l}
n \\
k
\end{array}\right) B_{k}(x) .
$$

On the other hand, we can rewrite (53) as

$$
P_{n}(x+1)+P_{n}(x)=\sum_{k=0}^{n}\left(\begin{array}{l}
n \\
k
\end{array}\right) P_{k}(x)+P_{n}(x) .
$$

The left-hand side of this equation is equal to $2 \mathcal{S}_{E}\left(P_{n}(x)\right)$, and so in the case that $P_{n}(x)=E_{n}(x)$, this leads to the formula $[8,24.5 .2]$

$$
\sum_{k=0}^{n}\left(\begin{array}{l}
n \\
k
\end{array}\right) E_{k}(x)+E_{n}(x)=2 x^{n}
$$

We can also let $h=-1$; this yields alternating versions of the formulae above. The result in (50) becomes

$$
P_{n}(x-1)-P_{n}(x)=\sum_{k=0}^{n-1}(-1)^{n-k}\left(\begin{array}{l}
n \\
k
\end{array}\right) P_{k}(x) .
$$

We repeat the derivation above to obtain an alternating version of the Bernoulli recurrence. We write

$$
\begin{aligned}
P_{n}(x-1)-P_{n}(x) & =-\int_{0}^{1} P_{n}^{\prime}(x-1+u) d u \\
& =-n \int_{0}^{1} P_{n-1}(x-1+u) d u
\end{aligned}
$$


Now let $g(u):=\chi_{[0,1]}$, so that the $P_{n}(x)$ are Bernoulli polynomials. Since

$$
-n \int_{0}^{1} B_{n-1}(x-1+u) d u=-n \mathcal{S}_{B}\left(B_{n-1}(x-1)\right)=-n(x-1)^{n-1},
$$

we can conclude that

$$
n(x-1)^{n-1}=\sum_{k=0}^{n-1}(-1)^{n-k+1}\left(\begin{array}{l}
n \\
k
\end{array}\right) B_{k}(x) .
$$

Alternatively, rewrite (53) as

$$
P_{n}(x-1)+P_{n}(x)=\sum_{k=0}^{n}(-1)^{n-k}\left(\begin{array}{l}
n \\
k
\end{array}\right) P_{k}(x)+P_{n}(x) .
$$

The left-hand side of this equation is equal to $2 \mathcal{S}_{E}\left(P_{n}(x-1)\right)$. Now suppose that we are in the Euler polynomial case, so that $P_{n}(x)=E_{n}(x)$. We thus obtain an alternating recurrence formula for the Euler polynomials:

$$
\sum_{k=0}^{n}(-1)^{n-k}\left(\begin{array}{l}
n \\
k
\end{array}\right) E_{k}(x)+E_{n}(x)=2(x-1)^{n} .
$$

Finally, we will prove a formula which simplifies proofs that will appear later in this paper. Set $x=0$ in (50) to get

$$
P_{n}(h)=\sum_{k=0}^{n}\left(\begin{array}{l}
n \\
k
\end{array}\right) P_{k}(0) h^{n-k} .
$$

We now introduce the definition of a Strodt number as $P_{k}:=P_{k}(0), k \geq 0$. Substituting $x$ for $h$ in (65) and then reindexing the sum, we get

$$
P_{n}(x)=\sum_{k=0}^{n}\left(\begin{array}{l}
n \\
k
\end{array}\right) P_{n-k} x^{k}
$$

This formula will be recalled in the next section to calculate a Strodt polynomial, and in Section 6 it will help us to prove an inversion formula for Strodt numbers.

4. STRODT POLYNOMIALS OF PROBABILITY DENSITIES. We have already seen how the generating functions and additive properties of Euler and Bernoulli polynomials can be recovered if one begins by defining them as Strodt polynomials. In fact, one could choose any density function and, proceeding in the same manner, obtain a class of polynomials with similar properties. In this section we will discover the Strodt polynomials associated with each of a selection of well-known probability distributions; the reader is encouraged to discover the consequences arising from other densities.

Example 4.1 (Gaussian Density Function). Here we take $g(u)=\frac{1}{\sqrt{\pi}} e^{-u^{2} / 2},-\infty<$ $u<\infty$. We calculate the moment generating function of this distribution:

$$
Q(t)=\frac{1}{\sqrt{\pi}} \int_{-\infty}^{\infty} e^{u t} e^{-u^{2} / 2} d u=e^{t^{2} / 4}
$$


The generating function for the Strodt polynomials in this case is thus

$$
\sum_{n=0}^{\infty} P_{n}(x) \frac{t^{n}}{n !}=\frac{e^{x t}}{Q(t)}=e^{x t-t^{2} / 4}
$$

At this point a similarity to the Hermite polynomials is evident; their generating function is known to be $e^{2 x t-t^{2}}[1,22.9 .17]$. Thus it follows that the Strodt polynomials in this case are scalings of the Hermite polynomials, as

$$
2^{n} P_{n}(x)=H_{n}(x) \quad \text { for all } n \in \mathbb{N} \text {. }
$$

We now have the following immediate corollary to Theorems 2.4 and 2.3.

Corollary 4.2. The Hermite polynomials $H_{n}(x)$ are scaled Strodt polynomials for the Gaussian density function. They satisfy

$$
\frac{1}{\sqrt{2 \pi}} \int_{-\infty}^{\infty} H_{n}(x+u) e^{-u^{2} / 2} d u=(2 x)^{n}, \quad \text { for all } n \in \mathbb{N}_{0},
$$

and

$$
\frac{d}{d x} H_{n}(x)=2 n H_{n-1}(x), \quad \text { for all } n \in \mathbb{N} \text {. }
$$

Proof. The $P_{n}(x)$ in (68) were contrived to satisfy the generating function that makes them the Strodt polynomials of the Gaussian distribution. Therefore they satisfy

$$
S_{g}\left(P_{n}(x)\right)=x^{n}
$$

which leads to (70), after one divides by a power of 2 . Similarly, (71) follows from Theorem 2.3.

The symmetry of the function $e^{-u^{2} / 2}$ about the origin leads to an additional property of the Strodt operator for this distribution.

Proposition 4.3. For $g(u)=\frac{1}{\sqrt{2 \pi}} e^{-u^{2} / 2}$, the operator $\mathcal{S}_{g}$ sends even polynomials to even polynomials and odd polynomials to odd polynomials.

Proof. Examine the image of the monomial $x^{n}$ under the Strodt operator. We expand $(x+u)^{n}$ in the integrand via the binomial theorem, yielding

$$
\mathcal{S}_{g}\left(x^{n}\right)=\frac{1}{\sqrt{2 \pi}} \sum_{j=0}^{n}\left(\begin{array}{l}
n \\
j
\end{array}\right) x^{j} \int_{-\infty}^{\infty} u^{n-j} e^{-u^{2} / 2} d u .
$$

We see that the integrals in this finite sum vanish if $n-j$ is an odd integer, for that causes the integrand to be an odd function. Therefore, the resulting polynomial must have the same parity as the original function, which is the parity of $n$ itself. Since any even (odd) polynomial is a linear combination of even (odd) monomials, the proposition follows by linearity of the operator. 
Remark 3. The Hermite polynomials are the only Appell sequence polynomials which are orthogonal; see [14] for a nice discussion of this topic. Since Strodt polynomials are a subset of Appell sequences, we know that Hermite polynomials are the only orthogonal Strodt polynomials as well.

Example 4.4 (Poisson Distribution). The Poisson distribution $X$ for a real parameter $\lambda$ is given by the probability function

$$
P(X=j)=e^{-\lambda} \frac{\lambda^{j}}{j !} \quad \text { for } j \in \mathbb{N}_{0} .
$$

This corresponds to the weight function

$$
g(u):=\sum_{j=0}^{\infty} \delta_{j}(u) e^{-\lambda} \frac{\lambda^{u}}{\Gamma(u+1)} .
$$

We will develop the main properties using a general value of $\lambda$ as far as this is possible.

Since the moment generating function is

$$
Q(t)=e^{-\lambda} \sum_{j=0}^{\infty} e^{j t} \frac{\lambda^{j}}{j !}=e^{\lambda\left(e^{t}-1\right)}
$$

the generating function for the Strodt polynomials is given by

$$
\sum_{n=0}^{\infty} P_{n, \lambda}(x) \frac{t^{n}}{n !}=e^{x t} e^{-\lambda\left(e^{t}-1\right)}
$$

where the polynomials also depend on the value of the real parameter $\lambda$. Letting $x=0$, we get

$$
\sum_{n=0}^{\infty} P_{n, \lambda} \frac{t^{n}}{n !}=e^{-\lambda\left(e^{t}-1\right)}
$$

where the coefficients on the left are Strodt numbers, which we define in a similar way as in the previous section: $P_{n, \lambda}:=P_{n, \lambda}(0)$. We expand the right-hand side of this equation in a Taylor series with respect to $t$ to obtain

$$
e^{-\lambda\left(e^{t}-1\right)}=\sum_{j=0}^{\infty} \sum_{n=0}^{\infty} \frac{j^{n} t^{n}}{n !} e^{\lambda} \frac{(-\lambda)^{j}}{j !}=\sum_{n=0}^{\infty} \frac{t^{n}}{n !} e^{\lambda} \sum_{j=0}^{\infty} \frac{j^{n}(-\lambda)^{j}}{j !} .
$$

In the inner sum, replace $j^{n}$ using the following well-known formula $[1,24.1 .4 \mathrm{~B}]$ :

$$
j^{n}=\sum_{k=0}^{n} S(n, k)(j)_{k}=\sum_{k=0}^{n} S(n, k) j(j-1) \cdots(j-k+1),
$$

where the $S(n, k)$ are the Stirling numbers of the second kind. These are defined as the number of ways to partition a set of $n$ elements into $k$ nonempty subsets; [11] describes their use in combinatorics. The above identity allows us to reach a closed form for the inner sum, and hence the Strodt numbers: 


$$
\begin{aligned}
P_{n, \lambda} & =e^{\lambda} \sum_{j=0}^{\infty} \frac{j^{n}(-\lambda)^{j}}{j !}=e^{\lambda} \sum_{k=0}^{n} S(n, k)(-\lambda)^{k} \sum_{j=k}^{\infty} \frac{(-\lambda)^{j-k}}{(j-k) !} \\
& =\sum_{k=0}^{n} S(n, k)(-\lambda)^{k} .
\end{aligned}
$$

The polynomial on the right is identified in [11] as the $m$ th Bell polynomial $B_{m}(-\lambda)$, and a similar derivation is given there. We will, however, eschew this notation in order to avoid confusion with the Bernoulli polynomials. Finally, we achieve a closed form for the Strodt polynomials. Recall (66), which says

$$
P_{n}(x)=\sum_{m=0}^{n}\left(\begin{array}{l}
n \\
m
\end{array}\right) P_{n-m} x^{m}=\sum_{m=0}^{n}\left(\begin{array}{l}
n \\
m
\end{array}\right) P_{m} x^{n-m} .
$$

Matching this with our derived closed form of the Strodt numbers, we obtain

$$
P_{n, \lambda}(x)=\sum_{m=0}^{n}\left(\begin{array}{l}
n \\
m
\end{array}\right) \sum_{k=0}^{m} S(m, k)(-\lambda)^{k} x^{n-m} .
$$

In the special cases $\lambda=-1$ and 1 , the Strodt numbers will reduce to the $m$ th Bell number and complementary Bell number [11], respectively.

Again, we have a corollary of Theorems 2.4 and 2.3.

Corollary 4.5. Let $P_{n, \lambda}(x)$ be defined by (83) for $n \in \mathbb{N}_{0}$ and fixed $\lambda \in \mathbb{R}$. Then

$$
e^{-\lambda} \sum_{j=0}^{\infty} P_{n, \lambda}(x+j) \frac{\lambda^{j}}{j !}=x^{n}
$$

and

$$
\frac{d}{d x} P_{n, \lambda}(x)=n P_{n-1, \lambda}(x)
$$

Proof. We derived (83) from the power series

$$
\sum_{n=0}^{\infty} P_{n, \lambda}(x) \frac{t^{n}}{n !}=e^{x t-\lambda\left(e^{t}-1\right)}=\frac{e^{x t}}{Q(t)},
$$

and thus by Theorem 2.4 the $P_{n, \lambda}(x)$ are the Strodt polynomials for the Poisson distribution with fixed parameter $\lambda$. Hence (84), which reads $\mathcal{S}_{g}\left(P_{n, \lambda}(x)\right)=x^{n}$, follows by definition of Strodt polynomials and (85) is a consequence of Theorem 2.3.

Example 4.6 (Exponential Distribution). For $\lambda>0$ we take the density function associated with the exponential distribution to be

$$
g(u):= \begin{cases}\lambda e^{-\lambda u} & \text { for } u>0 \\ 0 & u \leq 0\end{cases}
$$


and we calculate the moment generating function

$$
Q(t)=\lambda \int_{0}^{\infty} e^{u t-\lambda u} d u=\frac{\lambda}{\lambda-t}=\frac{1}{1-t / \lambda}
$$

for real $t<\lambda$. Therefore, by Theorem 2.4,

$$
\sum_{n=0}^{\infty} \mathcal{S}_{g}\left(x^{n}\right) \frac{t^{n}}{n !}=\frac{e^{x t}}{1-t / \lambda}
$$

We thus have the following property of the images of $x^{n}$ under the Strodt operator for the exponential distribution.

Proposition 4.7. When $g(u)$ is the exponential density (87), the Strodt operator $\mathcal{S}_{g}$ applied to the monomial $x^{n}$ gives a scaled Taylor series truncation of the exponential function:

$$
\mathcal{S}_{g}\left(x^{n}\right)=n ! \lambda^{-n} \sum_{m=0}^{n} \frac{(\lambda x)^{m}}{m !}, \text { for all } n \in \mathbb{N}_{0}, \lambda>0
$$

Proof. On the right-hand side of (89), expand $e^{x t}$ in a Taylor series and $(1-t / \lambda)^{-1}$ in a geometric series. Then match $t^{n}$ coefficients.

We claim that the Strodt polynomials for the exponential distribution are given by

$$
P_{0, \lambda}(x)=1, \quad P_{n, \lambda}(x)=x^{n}-\frac{n x^{n-1}}{\lambda} \quad \text { for } n \in \mathbb{N} .
$$

Indeed,

$$
\begin{aligned}
\mathcal{S}_{g}\left(x^{n}-\frac{n x^{n-1}}{\lambda}\right) & =\mathcal{S}_{g}\left(x^{n}\right)-\frac{n \mathcal{S}_{g}\left(x^{n-1}\right)}{\lambda} \\
& =n ! \lambda^{-n} \sum_{m=0}^{n} \frac{(\lambda x)^{m}}{m !}-n ! \lambda^{-n} \sum_{m=0}^{n-1} \frac{(\lambda x)^{m}}{m !} \\
& =x^{n}
\end{aligned}
$$

for $n \geq 1$, and $\mathcal{S}_{g}(1)=1$. The justification for (92) is the linearity of the Strodt operator, and (93) follows from (90). Note that one can calculate the Strodt polynomials much more directly by using (66). However, we include Proposition 4.7 for its inherent interest.

5. SUMMATION FORMULAE. The focus of Strodt's brief note [15] was to compare the summation formulae of Euler-Maclaurin and Boole. We now fill in the details for a general formula and show how it can be specified to obtain either formula.

We begin the argument for a general density function. Let $z=a+h$ for $0<h<1$. For a fixed integer $m \geq 0$, define the remainder as

$$
R_{m}(z):=f(z)-\sum_{k=0}^{m} \frac{\mathcal{S}_{g}\left(f^{(k)}\right)(a)}{k !} P_{k}(h)
$$


for a sufficiently smooth function $f$. The process of deriving a summation formula in general essentially reduces to finding an expression for $R_{m}(z)$ as an integral involving the Strodt polynomials corresponding to the operator $\mathcal{S}_{g}$.

Start with $m=0$. Since $P_{0}(z)=1$ and $\int g(u) d u=1$, we have

$$
\begin{aligned}
R_{0}(z) & =f(a+h)-\int f(a+u) g(u) d u \\
& =\int[f(a+h)-f(a+u)] g(u) d u .
\end{aligned}
$$

We rewrite the integrand in the right-hand side as

$$
f(a+h)-f(a+u)=\int_{u}^{h} f^{\prime}(a+s) d s,
$$

assuming that $f$ has a continuous derivative. Using Fubini's theorem, we switch the order of integration. This yields

$$
R_{0}(z)=\iint_{u}^{h} f^{\prime}(a+s) d s g(u) d u=\int V(s, h) f^{\prime}(a+s) d s,
$$

where we define the piecewise function

$$
V(s, h):= \begin{cases}\int_{-\infty}^{s} g(u) d u & \text { for } s<h, \\ \int_{-\infty}^{s} g(u) d u-1 & \text { for } s \geq h .\end{cases}
$$

At this point we separate the development into Euler-Maclaurin and Boole cases.

(a) For Boole summation, $P_{n}(x)=E_{n}(x)$ and $g(u)=\left(\delta_{0}(u)+\delta_{1}(u)\right) / 2$. We calculate that

$$
\begin{aligned}
2 \cdot V(s, h) & = \begin{cases}1 & \text { for } 0 \leq s<h \\
-1 & \text { for } h \leq s \leq 1 \\
0 & \text { otherwise }\end{cases} \\
& =\widetilde{E}_{0}(h-s) \chi_{[0,1]}(s),
\end{aligned}
$$

where $\widetilde{E}_{0}(x)$ is the periodic Euler polynomial on $[0,1]$. Thus we have

$$
f(a+h)=\mathcal{S}_{E}(f)(a)+\frac{1}{2} \int_{0}^{1} f^{\prime}(a+s) \tilde{E}_{0}(h-s) d s,
$$

which corresponds to (5) in the special case $m=1$ and $n=a+1$. We view this as the core formula in Boole summation, and the rest of (5) can be fleshed out by summing and integrating by parts.

We begin by rewriting (101) using the change of variables $x:=a+s$ in the integrand. Since $a \in \mathbb{N}, \widetilde{E}_{0}(a+h-s)=(-1)^{a} \widetilde{E}_{0}(h-s)$ and

$$
f(a+h)=\frac{1}{2}(f(a)+f(a+1))+\frac{(-1)^{a}}{2} \int_{a}^{a+1} f^{\prime}(x) \widetilde{E}_{0}(h-x) d x .
$$


Now replace $a$ in (102) with $j$ and take the alternating sum of both sides as $j$ ranges from $a$ to $n-1$. The sum telescopes and we combine the intervals of integration to obtain a single integral on $[a, n]$. This gives us

$$
\begin{aligned}
\sum_{j=a}^{n-1}(-1)^{j} f(j+h)= & \frac{1}{2}\left((-1)^{a} f(a)+(-1)^{n-1} f(n)\right) \\
& +\frac{1}{2} \int_{a}^{n} f^{\prime}(x) \tilde{E}_{0}(h-x) d x .
\end{aligned}
$$

This is exactly (5) with $m=1$.

To complete (5) for a general positive integer $m \geq 1$ requires a proof by induction. An integration by parts confirms that

$$
\begin{aligned}
\int_{a}^{n} f^{(k)}(x) \widetilde{E}_{k-1}(h-x) d s= & \frac{\widetilde{E}_{k}(h)}{k}\left((-1)^{n-1} f^{(k)}(n)+(-1)^{a} f^{(k)}(a)\right) \\
& +\frac{1}{k} \int_{a}^{n} \widetilde{E}_{k}(h-x) f^{(k+1)}(x) d x
\end{aligned}
$$

for $k \geq 0$, which supplies the induction step.

(b) Euler-Maclaurin summation (as in [15]): If we instead take $g(u)=\chi_{[0,1]}(u)$, then we write

$$
V(s, h)= \begin{cases}s & \text { for } 0 \leq s<h \\ s-1 & \text { for } h \leq s \leq 1 \\ 0 & \text { otherwise }\end{cases}
$$

Therefore,

$$
\begin{aligned}
R_{1}(z) & =\int_{0}^{1} V(s, h) f^{\prime}(a+s) d s-\int_{0}^{1} f^{\prime}(a+s) d s\left(h-\frac{1}{2}\right) \\
& =\int_{0}^{1} \widetilde{B}_{1}(s-h) f^{\prime}(a+s) d s .
\end{aligned}
$$

This is owing to the observation $V(s, h)-\left(h-\frac{1}{2}\right)=\widetilde{B}_{1}(s-h) g(s)$. Hence we have

$$
\begin{aligned}
f(a+h)= & \int_{0}^{1} f(s+a) d s+B_{1}(h)(f(a+1)-f(a)) \\
& +\int_{0}^{1} f^{\prime}(a+s) \widetilde{B}_{1}(s-h) d s .
\end{aligned}
$$

As in the Boole case, we are now essentially done. To completely recover (1), we simply integrate by parts and sum over consecutive integers.

Summing over the integers within the interval $[a, n-1]$ yields

$$
\begin{aligned}
\sum_{j=a}^{n-1} f(j+h)= & \int_{a}^{n} f(x) d x+B_{1}(h)(f(n)-f(a)) \\
& +\int_{a}^{n} f^{\prime}(x) \widetilde{B}_{1}(x-h) d x .
\end{aligned}
$$

May 2009] 
Here we have also shifted both intervals of integration via $x:=a+s$. This formula is the initial case for an induction proof. Also,

$$
\sum_{n=0}^{\infty} B_{n}(1-x) \frac{t^{n}}{n !}=\frac{t e^{(1-x) t}}{e^{t}-1}=\frac{t e^{-x t}}{1-e^{-t}}=\sum_{n=0}^{\infty} B_{n}(x) \frac{(-t)^{n}}{n !}
$$

whence we derive by matching coefficients the well-known fact $\widetilde{B}_{n}(-h)=$ $B_{n}(1-h)=(-1)^{n} B_{n}(h)$ for all $n \geq 0[1,23.1 .8]$. Now an integration by parts yields

$$
\begin{aligned}
\int_{a}^{n} f^{(k)}(x) \widetilde{B}_{k}(x-h) d x= & \frac{(-1)^{k+1} B_{k+1}(h)}{k+1}\left(f^{(k)}(n)-f^{(k)}(a)\right) \\
& -\frac{1}{k+1} \int_{a}^{n} f^{(k+1)}(x) \widetilde{B}_{k+1}(x) d x
\end{aligned}
$$

for all $k \geq 1$, which provides the induction step. The result is

$$
\begin{aligned}
\sum_{j=a}^{n-1} f(j+h)= & \int_{a}^{n} f(x) d x+\sum_{k=1}^{m} \frac{B_{k}(h)}{k !}\left(f^{(k-1)}(n)-f^{(k-1)}(a)\right) \\
& +\frac{(-1)^{m+1}}{m !} \int_{a}^{n} f^{(m)}(x) \widetilde{B}_{m}(x-h) d x
\end{aligned}
$$

This is a generalized version of (1), as one can see by taking the limit as $h \rightarrow 0$.

(c) The Taylor series approximation can be seen as another case in this general approach. Let $g(u)=\delta_{0}(u)$, so that $\mathcal{S}_{g}\left(x^{n}\right)=x^{n}$. This means that $P_{n}(x)=x^{n}$. We will call this operator $\mathcal{S}_{1}$, to be consistent with (6) as well as to indicate that this is the identity operator. In this case,

$$
V(s, h):= \begin{cases}1 & \text { for } 0<s<h \\ 0 & \text { otherwise }\end{cases}
$$

Thus we have

$$
f(a+h)=f(a)+\int_{a}^{a+h} f^{\prime}(x) d x .
$$

Here we have substituted $x:=a+s$ in the integral. We then use integration by parts to verify that

$$
\begin{aligned}
\int_{a}^{a+h}(x-a-h)^{k-1} f^{(k)}(x) d x= & -\frac{(-h)^{k}}{k} f^{(k)}(a) \\
& -\frac{1}{k} \int_{a}^{a+h}(x-a-h)^{k} f^{(k+1)}(x) d x
\end{aligned}
$$

for all $k \geq 1$. Therefore, by induction, for all $m \geq 0$,

$$
f(a+h)=\sum_{k=0}^{m} f^{(k)}(a) \frac{h^{k}}{k !}+\frac{(-1)^{m}}{m !} \int_{a}^{a+h}(x-a-h)^{m} f^{(m+1)}(x) d x .
$$


The derivations of the Euler-Maclaurin and Boole summation formulae, seen in this way, appear essentially similar to that of the Taylor series approximation. This comparison has been made in [7] in the Euler-Maclaurin and Taylor cases.

Remark 4. In each of the above cases, the proof relies on the fact that the function $V(s, h)$ can be related back to a polynomial from the original sequence. At present, we do not know how to extend this idea to a general Strodt polynomial.

6. INVERSION FORMULAE. In this section we use the Strodt numbers to generalize known inversion formulae for Bernoulli and Euler numbers. This generalization also allows us to recover such number sequences as the Bell numbers.

We begin by recalling a definition. For $n \in \mathbb{N}_{0}$, define the $n$th Strodt number as the constant coefficient of the $n$th Strodt polynomial; that is,

$$
P_{n}^{g}:=P_{n}^{g}(0) \text {. }
$$

As before, we will routinely omit the superscript $g$ in the notation. As a result of (47), the generating function for this sequence is

$$
\sum_{n=0}^{\infty} P_{n} \frac{t^{n}}{n !}=\frac{1}{Q(t)}
$$

where $Q(t)$ is as defined in (26).

We offer, as a motivation for these definitions, the apparent structure in the inversion formulas below [8, 24.5.9-10]. In each of the pairs of equations

$$
\begin{aligned}
a_{n} & =\sum_{k=0}^{n}\left(\begin{array}{l}
n \\
k
\end{array}\right) \frac{b_{n-k}}{k+1}, & b_{n} & =\sum_{k=0}^{n}\left(\begin{array}{l}
n \\
k
\end{array}\right) B_{k} a_{n-k} ; \\
a_{n} & =\sum_{k=0}^{\left\lfloor\frac{n}{2}\right\rfloor}\left(\begin{array}{c}
n \\
2 k
\end{array}\right) b_{n-2 k}, & b_{n} & =\sum_{k=0}^{\left\lfloor\frac{n}{2}\right\rfloor}\left(\begin{array}{c}
n \\
2 k
\end{array}\right) E_{2 k} a_{n-2 k} ;
\end{aligned}
$$

the set of equations on the left taken for all $n \in \mathbb{N}_{0}$ will imply those on the right for all $n$, and vice versa. The $B_{n}$ and $E_{n}$ denote the $n$th Bernoulli and Euler numbers, respectively. We can derive both these formulae from a general property of Strodt numbers.

Theorem 6.1. Let $P_{n}$ be the Strodt numbers associated with a probability measure $g(u)$ and $M_{k}$ its $k$ th moment as defined in (13). Then each formula of the pair

$$
a_{n}=\sum_{k=0}^{n}\left(\begin{array}{l}
n \\
k
\end{array}\right) b_{n-k} M_{k}, \quad b_{n}=\sum_{k=0}^{n}\left(\begin{array}{l}
n \\
k
\end{array}\right) a_{n-k} P_{k},
$$

taken for all $0 \leq n \leq m$, implies the other for all $0 \leq n \leq m$.

Proof. We show that each of the equations in (121) results from applying the Strodt operator to a polynomial.

Let $B(x):=\sum_{n=0}^{m}\left(\begin{array}{c}m \\ n\end{array}\right) b_{m-n} x^{n}$, and let $A(x)$ be defined by $A:=\mathcal{S}_{g}(B)$. By Proposition $2.2, A(x)$ is a degree- $m$ polynomial which can be represented by $A(x)=$ 
$\sum_{n=0}^{m}\left(\begin{array}{l}m \\ n\end{array}\right) a_{m-n} x^{n}$, where the $a_{m-n}$ are implicitly defined. We now solve for them. Recalling (12), if $f(x)=\sum_{n=0}^{m} f_{n} x^{n}$ and $h=\mathcal{S}_{g}(f)$, then

$$
h(x)=\sum_{n=0}^{m} h_{n} x^{n}
$$

where $h_{n}=\sum_{k=n}^{m} f_{k}\left(\begin{array}{l}k \\ n\end{array}\right) M_{k-n}$ and

$$
M_{l}:=\int u^{l} g(u) d u .
$$

With $B$ and $A$ in the place of $f$ and $g$, the result is

$$
\sum_{n=0}^{m}\left(\begin{array}{l}
m \\
n
\end{array}\right) a_{m-n} x^{n}=\sum_{n=0}^{m}\left(\sum_{k=n}^{m}\left(\begin{array}{l}
m \\
k
\end{array}\right) b_{m-k}\left(\begin{array}{l}
k \\
n
\end{array}\right) M_{k-n}\right) x^{n} .
$$

We then match $x^{n}$ coefficients, and use the combinatorial identity $\left(\begin{array}{l}m \\ k\end{array}\right)\left(\begin{array}{l}k \\ n\end{array}\right)=\left(\begin{array}{c}m \\ n\end{array}\right)\left(\begin{array}{c}m-n \\ k-n\end{array}\right)$ to reexpress the right-hand coefficient, so that

$$
\begin{aligned}
\left(\begin{array}{c}
m \\
n
\end{array}\right) a_{m-n} & =\left(\begin{array}{l}
m \\
n
\end{array}\right) \sum_{k=n}^{m}\left(\begin{array}{c}
m-n \\
k-n
\end{array}\right) b_{m-k} M_{k-n} \\
& =\left(\begin{array}{c}
m \\
n
\end{array}\right) \sum_{k=0}^{m-n}\left(\begin{array}{c}
m-n \\
k
\end{array}\right) b_{m-n-k} M_{k} .
\end{aligned}
$$

Therefore,

$$
a_{n}=\sum_{k=0}^{n}\left(\begin{array}{l}
n \\
k
\end{array}\right) b_{n-k} M_{k}, \quad 0 \leq n \leq m
$$

is equivalent to $A=\mathcal{S}_{g}(B)$, where

$$
A(x)=\sum_{k=0}^{n}\left(\begin{array}{l}
n \\
k
\end{array}\right) a_{n-k} x^{k} \quad \text { and } \quad B(x):=\sum_{k=0}^{n}\left(\begin{array}{l}
n \\
k
\end{array}\right) b_{n-k} x^{k} .
$$

This is the left-hand equation of (121).

For the second half, we write out $B=\mathcal{S}_{g}^{-1}(A)$ in detail:

$$
\mathcal{S}_{g}^{-1}(A(x))=\sum_{k=0}^{m}\left(\begin{array}{l}
m \\
k
\end{array}\right) a_{m-k} P_{k}(x) .
$$

Filling in the binomial formula for $P_{n}(x)$ from (66), we get:

$$
\mathcal{S}_{g}^{-1}(A(x))=\sum_{k=0}^{m}\left(\begin{array}{l}
m \\
k
\end{array}\right) a_{m-k} \sum_{n=0}^{k}\left(\begin{array}{l}
k \\
n
\end{array}\right) P_{k-n} x^{n} .
$$

Hence

$$
\sum_{n=0}^{m}\left(\begin{array}{l}
m \\
n
\end{array}\right) b_{m-n} x^{n}=\sum_{n=0}^{m}\left(\sum_{k=n}^{m}\left(\begin{array}{l}
m \\
k
\end{array}\right) a_{m-k}\left(\begin{array}{l}
k \\
n
\end{array}\right) P_{k-n}\right) x^{n}
$$


which is almost the same as (124), except that the $a_{n}$ and $b_{n}$ are switched, and we have $P_{k}$ in the place of $M_{k}$. Therefore, $B=\mathcal{S}_{g}^{-1}(A)$ must be equivalent to

$$
b_{n}=\sum_{k=0}^{n}\left(\begin{array}{l}
n \\
k
\end{array}\right) a_{n-k} P_{k}, \quad 0 \leq n \leq m,
$$

which is the right-hand equation of (121).

We conclude that the set of these equations for $0 \leq n \leq m$ is equivalent to (126) via $A=\mathcal{S}_{g}(B)$.

The Bernoulli numbers are given in $[1,23.1 .2]$ by the evaluation $B_{l}:=B_{l}(0)$. Thus they are the Strodt numbers for the Bernoulli operator. The moment $M_{k}$ is thus calculated as

$$
M_{k}=\int_{0}^{1} u^{k} d u=\frac{1}{k+1},
$$

and (119) is recovered immediately from this and (121).

To recover the corresponding equation for Euler numbers, we consider the polynomials $\mathcal{E}_{n}(x)$ that are generated by the distribution $g(u)=\frac{1}{2}\left(\delta_{-1}(u)+\delta_{1}(u)\right)$. This distribution is a scaling of the distribution in the Euler polynomial case, as it averages the points -1 and 1 as opposed to 0 and 1 . The generating function of the $\mathcal{E}_{n}(x)$ is

$$
\sum_{n=0}^{\infty} \mathcal{E}_{n}(x) \frac{t^{n}}{n !}=\frac{2 e^{x t}}{e^{-t}+e^{t}}
$$

as one can check by using Theorem 2.4 and calculating

$$
Q(t)=\frac{e^{(-1) \cdot t}+e^{1 \cdot t}}{2}
$$

The Strodt numbers corresponding to this distribution, $\mathcal{E}_{n}:=\mathcal{E}_{n}(0)$, are generated by

$$
\sum_{n=0}^{\infty} \mathcal{E}_{n} \frac{t^{n}}{n !}=\frac{2}{e^{-t}+e^{t}}=\operatorname{sech}(t)
$$

This, however, is exactly the generating function for Euler numbers [8, 24.2.6], and so $\mathcal{E}_{n}=E_{n}$ for all $n \geq 0$. At this point we also see (by the evenness of the hyperbolic secant function) that the odd Euler numbers are equal to zero. The $k$ th moment of this function is calculated as

$$
\int u^{k} g(u) d u=\frac{1^{k}+(-1)^{k}}{2}
$$

and the pair in (121) become

$$
a_{n}=\sum_{k=0}^{n}\left(\begin{array}{l}
n \\
k
\end{array}\right) b_{n-k}\left(\frac{1^{k}+(-1)^{k}}{2}\right) \quad \text { and } \quad b_{n}=\sum_{k=0}^{n}\left(\begin{array}{l}
n \\
k
\end{array}\right) a_{n-k} E_{k} .
$$

Now we verify that the odd terms in both of the above sums are equal to zero, the left because of vanishing odd moments, and the right because of vanishing odd Euler numbers. The result is (120). 
Now that we have seen a uniform derivation of the inversion formulae for both Euler and Bernoulli numbers, we will develop a less obviously connected formula that is nonetheless a consequence of Proposition 6.1. We return to Example 4.4 in Section 4 , where we calculated

$$
P_{n, \lambda}(x)=\sum_{m=0}^{n}\left(\begin{array}{l}
n \\
m
\end{array}\right) \sum_{j=0}^{m} S(m, j)(-\lambda)^{j} x^{n-m},
$$

the Strodt polynomials generated by the Poisson measure,

$$
g(u)=\sum_{j=0}^{\infty} \frac{\delta_{j}(u) e^{-\lambda} \lambda^{u}}{\Gamma(u+1)} .
$$

Evaluating at $x=0$, we find that the Strodt number sequence is given by

$$
P_{n}(\lambda)=P_{n, \lambda}(0)=\sum_{j=0}^{n} S(n, j)(-\lambda)^{j}
$$

which are Bell polynomials [11]. Computing the $k$ th moment of the Poisson distribution we obtain Bell polynomials again:

$$
M_{k}(\lambda)=e^{\lambda} \sum_{j=0}^{\infty} \frac{j^{k} \lambda^{j}}{j !}=\sum_{j=0}^{k} S(n, j) \lambda^{j} .
$$

The derivation of this identity is contained in Example 4.4. Now, employing (121), we derive

$$
a_{n}=\sum_{k=0}^{n}\left(\begin{array}{l}
n \\
k
\end{array}\right) b_{n-k}\left(\sum_{j=0}^{k} S(n, j) \lambda^{j}\right)
$$

and

$$
b_{n}=\sum_{k=0}^{n}\left(\begin{array}{l}
n \\
k
\end{array}\right) a_{n-k}\left(\sum_{j=0}^{k} S(n, j)(-\lambda)^{j}\right),
$$

as a new pair of inversion formulae. When $\lambda=1$, the moments become sums across rows in the triangle of Stirling numbers, and the Strodt numbers become alternating sums of the same. These are known [11] as Bell numbers, $\mathcal{B}_{n}$, and complementary Bell numbers, $\widetilde{\mathcal{B}}_{n}$, respectively. Therefore, each of the pair

$$
a_{n}=\sum_{k=0}^{n}\left(\begin{array}{l}
n \\
k
\end{array}\right) b_{n-k} \mathcal{B}_{k} \quad \text { and } \quad b_{n}=\sum_{k=0}^{n}\left(\begin{array}{l}
n \\
k
\end{array}\right) a_{n-k} \widetilde{\mathcal{B}}_{k},
$$

implies the other.

7. ASYMPTOTIC PROPERTIES. In [8] we find asymptotic formulae for Bernoulli and Euler polynomials as $n \rightarrow \infty$. Specifically,

$$
(-1)^{\lfloor n / 2\rfloor-1} \frac{(2 \pi)^{n}}{2(n !)} B_{n}(x) \rightarrow \begin{cases}\cos (2 \pi x), & n \text { even, } \\ \sin (2 \pi x), & n \text { odd }\end{cases}
$$


and

$$
(-1)^{\lfloor(n+1) / 2\rfloor} \frac{\pi^{n+1}}{4(n !)} E_{n}(x) \rightarrow \begin{cases}\sin (\pi x), & n \text { even, } \\ \cos (\pi x), & n \text { odd },\end{cases}
$$

appear as 24.11.5 and 24.11.6. The convergence is uniform in $x$ on compact subsets of $\mathbb{C}$.

Experimenting with plots for real $x$ as $n$ becomes increasingly large suggests that a similar asymptotic property is true for any Strodt uniform interpolation polynomial $P_{n}(x)$. As an example of our experimental results, Figure 1 displays the Strodt polynomials $P_{n}(x)$ for $g(u)=\frac{1}{3}\left(\delta_{0}(u)+\delta_{1 / 2}(u)+\delta_{1}(u)\right)$, which is the 3-point mean. We have displayed $n=20$ and $n=40$ for the Strodt polynomials, multiplied by a conjectured scaling factor $(-1)^{\lfloor n / 2\rfloor-1} 2^{2 n} \pi^{n+1} /\left(n ! 3^{n+3 / 2}\right)$ and horizontally offset by $1 / 4$. They clearly appear to be converging to $\cos \frac{4 \pi}{3} x$.

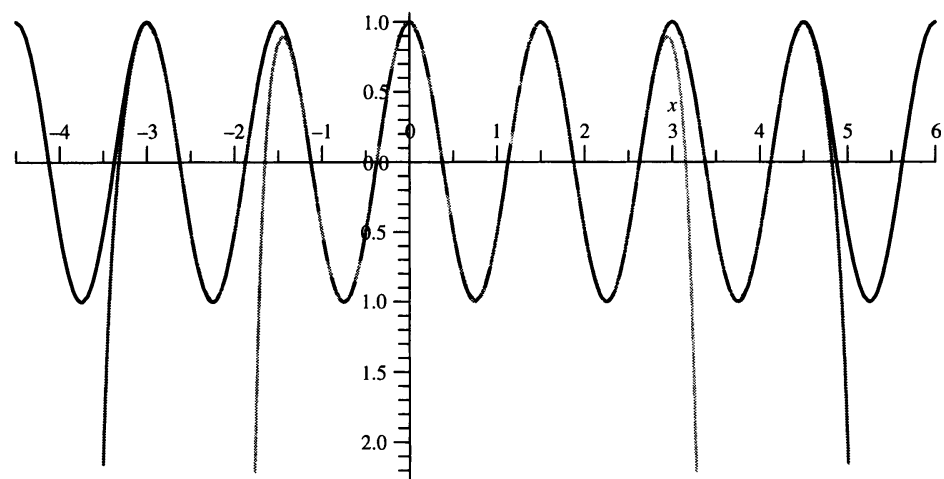

Figure 1. Graph of $\cos \frac{4 \pi}{3} x$ versus $(-1)^{\lfloor n / 2\rfloor-1} \frac{2^{2 n} \pi^{n+1}}{n ! 3^{n+3 / 2}} P_{n}(x-1 / 4)$ for $n=20$ and $n=40$.

The general situation is this: The uniform Strodt polynomials $P_{n}^{m}(x)$ on $m$ points have generating function

$$
\sum_{n \geq 0} P_{n}^{m}(x) \frac{t^{n}}{n !}=\frac{m e^{x t}}{\sum_{j=0}^{m-1} \exp \frac{j t}{m-1}}
$$

For $m=2$ this generates the Euler polynomials. We used experimental evidence to formulate a theorem for $m$-point asymptotics. Note that the the piecewise formulation above is unnecessary; for example, (142) could instead be written as

$$
\frac{(2 \pi)^{n}}{2(n !)} B_{n}(x) \sim \cos \left(2 \pi x+\frac{n \pi}{2}+\pi\right) .
$$

This is the notation that we will use below.

By experimenting with graphs of Strodt polynomials multiplied by scaling factors, we conjectured that for all $m \geq 2$, there are constants $C_{m}$ such that, as $n \rightarrow \infty$, we have

$$
C_{m} \frac{\pi^{n+1}}{n !}\left(\frac{2 m-2}{m}\right)^{n} P_{n}^{m}(x) \sim \cos \left(\frac{2 m-2}{m} \pi x+\frac{n \pi}{2}\right)
$$


The next step was to discover the closed form of the constants $C_{m}$. We did this by first calculating values of the approximants $P_{2 n}^{m}(0)$ for large values of $n$. This suggested exact values for $C_{m}, 2 \leq m \leq 16$, which were algebraic and appeared to be simpler for $m$ even. Then we used mathematical software to hunt for the polynomial of minimal degree which yielded zero when evaluated at $C_{m}$. From the first few polynomials-and associated radicals - plus some further numerical values, it seemed that the constants were trigonometric in origin. Indeed, we concluded that $C_{m} \rightarrow 1 /(2 \pi)$ and that

$$
C_{m}=\frac{\csc \left(\frac{\pi}{m}\right)}{2 m} \text {. }
$$

The former follows from the latter. We now restate the theorem and prove it.

Theorem 7.1. Let $x \in \mathbb{R}$. For all $m \geq 2$, as $n \rightarrow \infty$, we have

$$
\frac{\csc \left(\frac{\pi}{m}\right)}{2 m} \frac{\pi^{n+1}}{n !}\left(\frac{2 m-2}{m}\right)^{n} P_{n}^{m}(x) \sim \cos \left(\frac{2 m-2}{m} \pi x+\frac{n \pi}{2}\right) .
$$

Proof. The geometric sum in the denominator of (144) can be rewritten so that the generating function for the $m$-point Strodt polynomials is seen as $m e^{x t}\left(e^{\frac{t}{m-1}}-1\right) /$ $\left(e^{\frac{m t}{m-1}}-1\right)$. Now make the changes of variables $x \leftarrow x-\frac{1}{2 m-2}$ and $t \leftarrow \pi i t$ in the generating function, leading to

$$
\sum_{n \geq 0} P_{n}^{m}\left(x-\frac{1}{2 m-2}\right) \frac{(\pi i t)^{n}}{n !}=\frac{m e^{i \pi x t} e^{-i \frac{\pi t}{2 m-2}}\left(e^{i \frac{\pi t}{m-1}}-1\right)}{e^{i \frac{m \pi t}{m-1}}-1} .
$$

Then substitute $z=\frac{m t}{2 m-2}$ to obtain

$$
\sum_{n \geq 0} P_{n}^{m}\left(x-\frac{1}{2 m-2}\right) \frac{(\pi i)^{n}}{n !}\left(\frac{2 m-2}{m}\right)^{n} z^{n}=m e^{i \pi\left(\frac{2 m-2}{m} x-1\right) z} \frac{\sin \frac{\pi z}{m}}{\sin \pi z} .
$$

Finally, shift back $x \rightarrow x-\frac{1}{2 m-2}$ and separate the formula into real and imaginary parts to get generating functions for even and odd Strodt polynomials,

$$
\sum_{n \geq 0} \frac{(-1)^{n} \pi^{2 n}}{(2 n) !}\left(\frac{2 m-2}{m}\right)^{2 n} P_{2 n}^{m}(x) z^{2 n}=\frac{m \cos \left(\pi \frac{2 m-2}{m} x z\right) \sin \frac{\pi z}{m}}{\sin \pi z}
$$

and

$$
\sum_{n \geq 0} \frac{(-1)^{n+1} \pi^{2 n+1}}{(2 n+1) !}\left(\frac{2 m-2}{m}\right)^{2 n+1} P_{2 n+1}^{m}(x) z^{2 n+1}=\frac{m \sin \left(\pi \frac{2 m-2}{m} x z\right) \sin \frac{\pi z}{m}}{\sin \pi z} .
$$

We wish to extract the asymptotic formulae directly from these two generating functions. We will show the details for the even case; the odd will be similar. We use Darboux's Method to obtain the asymptotics. In order to do this, we must find a comparison function $G(z):=\sum g_{n} x^{n}$ to

$$
F(z):=\frac{m \cos \left(\pi \frac{2 m-2}{m} x z\right) \sin \frac{\pi z}{m}}{\sin \pi z}
$$


such that the difference $F-G$ is continuous on $|z| \leq r$, where $r$ is the smallest radius containing singularities of $F(z)$. We also wish for the asymptotics of $g_{n}$ to be known. A result due to Darboux (and discussed in detail in [10]) then proves that

$$
f_{n} \sim g_{n}+o\left(r^{-n} n^{-k}\right)
$$

if $F-G$ is $k$ times continuously differentiable.

In this case, $r=1$, as the generating function $F$ has singularities at $z= \pm 1$. An appropriate choice of comparison function is $G(z)=\frac{2 m}{\pi} \sin \frac{\pi}{m} \cos \left(\frac{2 m-2}{m} \pi x\right) /\left(1-z^{2}\right)$, as

$$
\begin{aligned}
\lim _{z \rightarrow 1^{-}}\left(1-z^{2}\right) F(z) & =\lim _{z \rightarrow-1^{+}}\left(1-z^{2}\right) F(z) \\
& =\frac{2 m}{\pi} \cos \left(\frac{2 m-2}{m} \pi x\right) \sin \frac{\pi}{m}
\end{aligned}
$$

and

$$
g_{2 n}=\frac{2 m}{\pi} \cos \left(\frac{2 m-2}{m} \pi x\right) \sin \frac{\pi}{m}
$$

Now $F-G$ has removable singularities at $z= \pm 1$ and can thus be analytically continued to the unit disk. Therefore, for fixed $m$ and $x$,

$$
\frac{(-1)^{n} \pi^{2 n}}{(2 n) !}\left(\frac{2 m-2}{m}\right)^{2 n} P_{2 n}^{m}(x) \sim \frac{2 m}{\pi} \cos \left(\frac{2 m-2}{m} \pi x\right) \sin \frac{\pi}{m}+o\left(n^{-k}\right)
$$

for any $k \in \mathbb{N}$. This can be directly matched to the conjectures (148) and (147). The proof for the odd asymptotics is similar, but with $G(z)=\frac{2 m z}{\pi} \sin \frac{\pi}{m} \sin \left(\frac{2 m-2}{m} \pi x\right) /$ $\left(1-z^{2}\right)$.

Remark 5. For $m=2$ and in the limit as $m \rightarrow \infty$ we recover known asymptotics for Euler and Bernoulli polynomials, respectively. The error in (156) is uniform; that is, its order does not depend on $m$ or $x$. This justifies our letting $m \rightarrow \infty$ to recover the asymptotic formulae for Bernoulli polynomials.

Remark 6. We do not yet have a conjecture for the precise asymptotic formula for general Strodt polynomials. Our preliminary experiments suggest there will be similarly interesting results for continuous probability densities.

8. CONCLUSIONS. Our project started with the goal of elucidating Strodt's idea of using integral operators to derive the Euler-Maclaurin and Boole summation formulae. Using Strodt operators to define the Bernoulli and Euler polynomials, we have ultimately suggested, largely by example, the variety of properties that can be recovered. This lends some important perspective. We see their properties not just as two isolated cases, but as part of a general context. They are Strodt polynomials, which we have defined as a special class of Appell sequences that satisfy a property involving Strodt operators. We see the worth of this additional property in the proofs of the summation and inversion formulae, and it may well assist in describing the asymptotics. Overall, the description of these polynomials by their images under integral operators enhances the connectivity of seemingly unrelated table identities. 
ACKNOWLEDGMENTS. The third author acknowledges partial support from the AARMS Director's Postdoctoral Fellowship.

\section{REFERENCES}

1. M. Abramowitz and I. Stegun, Handbook of Mathematical Functions with Formulas, Dover, New York, 1972.

2. G. Andrews, R. Askey, and R. Roy, Special Functions, Encyclopedia of Mathematics and its Applications, vol. 71, Cambridge University Press, New York, 1999.

3. B. Berndt, Character analogues of the Poisson and Euler-Maclaurin summation formulas with applications, J. Number Theory 7 (1975) 413-445.

4. G. Boole, A Treatise on the Calculus of Finite Differences, 2nd ed., Dover, New York, 1960, reprint of Macmillan, London, 1872.

5. J. Borwein, P. Borwein, and K. Dilcher, Pi, Euler numbers, and asymptotic expansions, this MONTHLY 96 (1989) 681-687.

6. W. Feller, An Introduction to Probability Theory and Its Applications, vol. I-II, Wiley, New York, 1968.

7. V. Lampret, The Euler-Maclaurin and Taylor formulas: Twin, elementary derivations, Math. Mag. 74 (2001) 109-122.

8. NIST, Digital Library of Mathematical Functions, available at http://dlmf .nist .gov/ (forthcoming).

9. N. E. Nôrlund, Vorlesungen über Differenzenrechnung, Springer-Verlag, Berlin, 1924.

10. F. W. J. Olver, Asymptotics and Special Functions, Academic Press, New York, 1974.

11. J. Riordan, An Introduction to Combinatorial Analysis, Wiley, New York, 1958.

12. S. Roman, The Umbral Calculus, Academic Press, New York, 1984.

13. H. Royden, Real Analysis, 3rd ed., Prentice Hall, Englewood Cliffs, NJ, 1988.

14. J. Shohat, The relation of classical orthogonal polynomials to the polynomials of Appell, Amer. J. Math. 58 (1936) 453-464.

15. W. Strodt, Remarks of the Euler-Maclaurin and Boole summation formulas, this MoNTHLY 67 (1960) $452-454$.

16. K. Stromberg, An Introduction to Classical Real Analysis, Wadsworth, Belmont, CA, 1981.

17. N. Temme, Special Functions, An Introduction to the Classical Functions of Mathematical Physics, Wiley, New York, 1996.

18. Wikipedia, "Bernoulli Polynomials," available at http://en.wikipedia.org/wiki/Bernoulli_ Polynomials.

JONATHAN M. BORWEIN is currently Canada Research Chair in Collaborative Technology at Dalhousie University. His primary current interest is in computer-assisted discovery in mathematics. He is a former President of the Canadian Mathematical Society, a Fellow of the Royal Society of Canada and of the AAAS, a Foreign Member of the Bulgarian Academy of Science, and a former co-recipient of the Chauvenet prize.

Faculty of Computer Science, Dalhousie University, Halifax, NS Canada B3H 4R2

jborwein@cs.dal.ca

NEIL J. CALKIN is associate professor at Clemson University, in the Department of Mathematical Sciences, an AMS, MAA, and SIAM member, and co-founder and past Managing Editor of the Electronic Journal of Combinatorics. His main research interests are in combinatorial and probabilistic methods, particularly in number theory. He discovered Strodt's note, in electronic version, by typing "Boole summation" into a popular search engine.

Department of Mathematical Sciences, Clemson University, Clemson, SC 29634

calkin@clemson.edu

DANTE MANNA is currently a Postdoctoral fellow at Dalhousie University, studying classical analysis. He earned a B.A. in Mathematics at Wesleyan University in 2001, and his $2006 \mathrm{Ph}$.D. in Mathematics from Tulane University was among the first awarded in post-Katrina New Orleans. His future in mathematics was possibly foreshadowed by winning "Class Contrarian" in high school, although he did not realize his passion for the subject until, ironically, taking a semester of intensive (Mandarin) Chinese Language study at Associated Colleges in China in Beijing.

Department of Mathematics and Statistics, Dalhousie University, Halifax, NS Canada B3K IW5

dmanna@mathstat.dal.ca 